\title{
Energy-Resolved Positron Annihilation in Flight in Solid Targets
}

\section{Citation}

Weber, M. H., A. W. Hunt, J. A. Golovchenko, and K. G. Lynn. 1999. Energy-Resolved Positron Annihilation in Flight in Solid Targets. Physical Review Letters 83, no. 22: 4658-4661. doi:10.1103/physrevlett.83.4658.

\section{Published Version}

doi:10.1103/PhysRevLett.83.4658

\section{Permanent link}

http://nrs.harvard.edu/urn-3:HUL.InstRepos:29405825

\section{Terms of Use}

This article was downloaded from Harvard University's DASH repository, and is made available under the terms and conditions applicable to Other Posted Material, as set forth at http:// nrs.harvard.edu/urn-3:HUL.InstRepos:dash.current.terms-of-use\#LAA

\section{Share Your Story}

The Harvard community has made this article openly available.

Please share how this access benefits you. Submit a story.

\section{Accessibility}




\title{
Energy-Resolved Positron Annihilation in Flight in Solid Targets
}

\author{
M. H. Weber, ${ }^{1}$ A. W. Hunt, ${ }^{2}$ J. A. Golovchenko, ${ }^{2,3}$ and K. G. Lynn ${ }^{1}$ \\ ${ }^{1}$ Department of Physics, Washington State University, Pullman, Washington 99164 \\ ${ }^{2}$ Department of Physics, Harvard University, Cambridge, Massachusetts 02138 \\ ${ }^{3}$ Rowland Institute for Science, Cambridge, Massachusetts 02142
}

(Received 28 June 1999)

\begin{abstract}
Energy-resolved two-quantum annihilation in flight of positrons with energies ranging from 10 to $71.6 \mathrm{keV}$ was observed. An energy-dispersive two-detector coincidence system was used to observe the sum and difference energies of the $\gamma$ rays from annihilating positron-electron pairs. For positrons penetrating carbon foils the $c / v$ dependence of the annihilation cross section is confirmed. Spectra obtained from gold-coated carbon foils show evidence of in-flight annihilation with gold $M$-shell electrons.
\end{abstract}

PACS numbers: 78.70.Bj, 14.60.-z, 24.80. $+\mathrm{y}, 25.30 . \mathrm{Hm}$

As energetic positrons penetrate a solid target they rapidly loose kinetic energy via electronic collisions to several $\mathrm{eV}$ within $\sim 0.1 \mathrm{ps}[1]$ and come into thermal equilibrium with the target within 10 ps [2]. The characteristic lifetime of the positron in a solid target is several 100 ps which is consistent with the observation that virtually all positrons annihilate with thermal kinetic energy. There is, however, a finite probability of annihilation during the slowing down process. Two-quantum annihilation in flight (TQAF) has been observed for $400 \mathrm{keV}$ to $\mathrm{MeV}$ energy positrons in solids [3-8]. For low $Z$ targets the results generally agree well with theoretical energy integrated cross sections. Small deviations were explained with experimental uncertainties in target thickness, the energy distribution of the positron beam, and the coincidence schemes available at the time. For high $Z$ targets the experimental results exceed QED predictions. Kilian suggested that contributions from single-quantum annihilation events are the reason [8]. Thirty years later, interest in TQAF revived briefly during a search for anomalies in the electron-positron scattering cross section near the $Z^{0}$ mass in the $\mathrm{GeV}$ range [9-11]. More recently investigators returned to in-flight annihilation in the low $\mathrm{MeV}$ region after sharp electron-positron pair sum energy peaks were observed and postulated to be the signature of a new particle [12-14].

TQAF contributes only a tiny fraction to the total annihilation spectrum in thick targets and is ignored when research with thermalized positrons concentrates on solid state phenomena. Annihilation spectroscopy based on the Doppler broadening of the annihilation line centered at $m_{0} c^{2}=511 \mathrm{keV}$ is a useful tool to obtain electron momentum distributions in single crystal solids and near defects [15]. The combination of a monochromatic variable-energy positron beam with the coincident observation of both annihilation quanta $[16,17]$ permits the measurement of the total energy of the electron-positron pair with high energy resolution and excellent signal to noise ratio. We report on energy resolved measurements of TQAF of positrons with incident energies from 10 to $70 \mathrm{keV}$. The relative cross section for annihilation in flight as a function of positron energy was measured and compared with the predicted $c / v$ dependence. In a gold target TQAF with $M$-shell core electrons was observed.

Two detectors register the energies $E_{1}$ and $E_{2}$ of the two photons from an annihilation event in coincidence. Their sum $\sum E$ is the sum of the rest masses of the electron and the positron $2 m_{0} c^{2}$, the kinetic energy of the positron $T$, and any electron binding energy $E_{b}$ :

$$
\sum E=E_{1}+E_{2}=2 m_{0} c^{2}+T-E_{b} .
$$

In standard Doppler broadening measurements with thermalized positrons $T \ll E_{b}$. Here the opposite is the case. The kinetics of the annihilation process determine the difference in energy of the detected photons; $\Delta E=$ $E_{1}-E_{2}$ is a function of the angle between the positron momentum $\vec{p}$ and the photon velocity $\vec{c}$,

$$
\Delta E=\frac{\vec{c} \cdot \vec{p}-\vec{c}^{2} \vec{p}^{2} / \sum E}{1-\vec{c} \cdot \vec{p} / \sum E}
$$

The value of $\Delta E$ is largest when the photons emerge parallel and antiparallel to the positron direction $\left(\Delta E_{\max }=\right.$ $\pm 276 \mathrm{keV}$ for $T=70 \mathrm{keV})$. Dirac [18] calculated the cross section for TQAF $\Phi_{\mathrm{TQAF}}$ between an electron at rest and a positron of total energy $E_{+}$[Eq. (3)].

$$
\Phi_{\mathrm{TQAF}}=\pi r_{0}^{2} \cdot \frac{1}{\gamma+1}\left[\frac{\gamma^{2}+4 \gamma+1}{\gamma^{2}-1} \ln \left(\gamma+\sqrt{\gamma^{2}-1}\right)-\frac{y+3}{\sqrt{y^{2}-1}}\right], \quad \gamma=\frac{E_{+}}{m_{0} c^{2}} .
$$

The equation contains the classical electron radius $r_{0}$ and the ratio $\gamma$ of the total energy of the positron to its rest mass. For small velocities $v / c<1$ the cross section becomes proportional to $c / v$, the inverse of the positron velocity. This is the case for all energies considered in this work. 
We investigate TQAF in the energy range below $72 \mathrm{keV}$, which previously could not be distinguished from thermalized positron annihilations. A $50 \mathrm{~nm}$ thick carbon foil was used as the target. This thickness is close to the ideal thickness, where only one inelastic process occurs. A second $50 \mathrm{~nm} \mathrm{C}$ foil was sputter coated with $\mathrm{Au}$ for $200 \mathrm{~s}$ to an estimated thickness of $50 \mathrm{~nm}$. A monoenergetic positron beam with an intensity of $\sim 5 \times$ $10^{4} e^{+} / \mathrm{s}$ was guided magnetically to the target. Over $90 \%$ of the positrons are transmitted and were guided to a shielded beam dump. Magnetic bending fields prevented backscattered positrons from returning to the target.

Two germanium detectors with $1.65 \mathrm{keV}$ FWHM energy resolution at $514 \mathrm{keV}$ were positioned $46 \mathrm{~mm}$ apart symmetrically on either side of the target on a line perpendicular to the incident beam. Both annihilation photons were detected in coincidence and collected in a two-dimensional histogram as a function of the difference of their energies $\Delta E$ ( $x$ axis) and their sum $\sum E$. An example is shown in Fig. 1 for the case of $71.6 \mathrm{keV}$ positrons impinging on the $\mathrm{Au}$ coated $\mathrm{C}$ foil. The $\log _{10}$ of the counts in each bin of the histogram are coded in a grey scale from white corresponding to 0 or 1 via dark grey and light grey count to black for $10^{2}$ counts. More than 100 events per bin were also plotted in black.

Annihilations of thermalized positrons dominate the spectrum at $\left[\sum E, \Delta E\right]=[1022,0] \mathrm{keV}$. Coincidences of a $511 \mathrm{keV} \gamma$-ray event in one detector with an incomplete charge collection or pileup event in the other cause the diagonal "ridges." Compton scattering from one detector to the other produces the smaller ridge along lines of constant $\sum E=1022 \mathrm{keV}$. Photons of $1.46 \mathrm{MeV}$ from ${ }^{40} \mathrm{~K}$ can trigger a coincidence by Compton scattering from one detector to the other. These events and thermal positron annihilations allow for an independent calibration

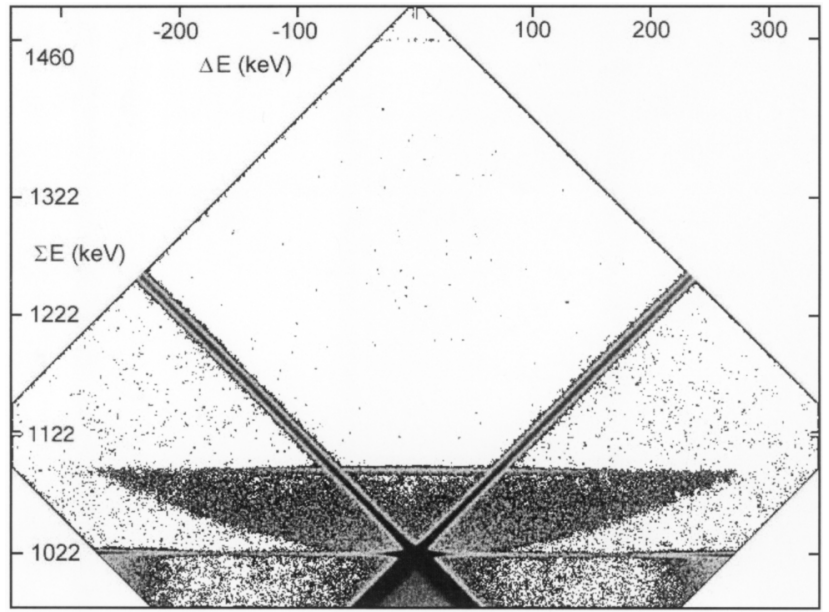

FIG. 1. 2D histogram of TQAF data from Au sputtered onto a $\mathrm{C}$ foil spanned by $\Delta E$ ( $x$ axis) and $\sum E$. Counts are color coded from $\leq 1$ (white) to $10^{2}$ and larger (black) per bin on a $\log _{10}$ scale. To enhance features with few counts all bins with more than 100 counts were set to 100 . of the sum energy scale. The line with constant sum energy $\sum E=1093.6 \mathrm{keV}$ is due to TQAF. It precisely matches the incident positron beam energy of $71.6 \mathrm{keV}$ and shifts with the incident beam energy. Some positrons will scatter inelastically and then annihilate. These events appear as a "bowl"-shaped region between the thermal peak $\left(\sum E=1022 \mathrm{keV}\right)$ and the TQAF line.

To analyze the TQAF data the ridges are eliminated by replacing them with fits through the baseline for each $\sum E$. Cuts are performed on the $2 \mathrm{D}$ histogram to show TQAF as a function of $\Delta E$ ( $\Delta E$ spectrum) and $\sum E$ ( $\sum E$ spectrum). The latter consists of an integration along $\Delta E$ over the full range of $\pm 350 \mathrm{keV}$. The $\Delta E$ spectrum is an integration of a $10 \mathrm{keV}$ wide window along $\sum E$, centered at $\sum E=T+2 m_{0} c^{2}$. Example cuts from the gold-coated carbon foil data are shown in Figs. 2(a) and 2(b) for the $\Delta E$ and the $\sum E$ spectra, respectively.

The width of the TQAF peak in the $\sum E$ spectrum is $4.3 \pm 0.2 \mathrm{keV}$ for the gold-coated foil. A broad spectrum appears in the $\Delta E$ spectrum. A calculation of the $\Delta E$ spectrum based on Eq. (2) and the angular dependence of the detection efficiency is also shown and agrees well with the central broad peak of the spectrum.

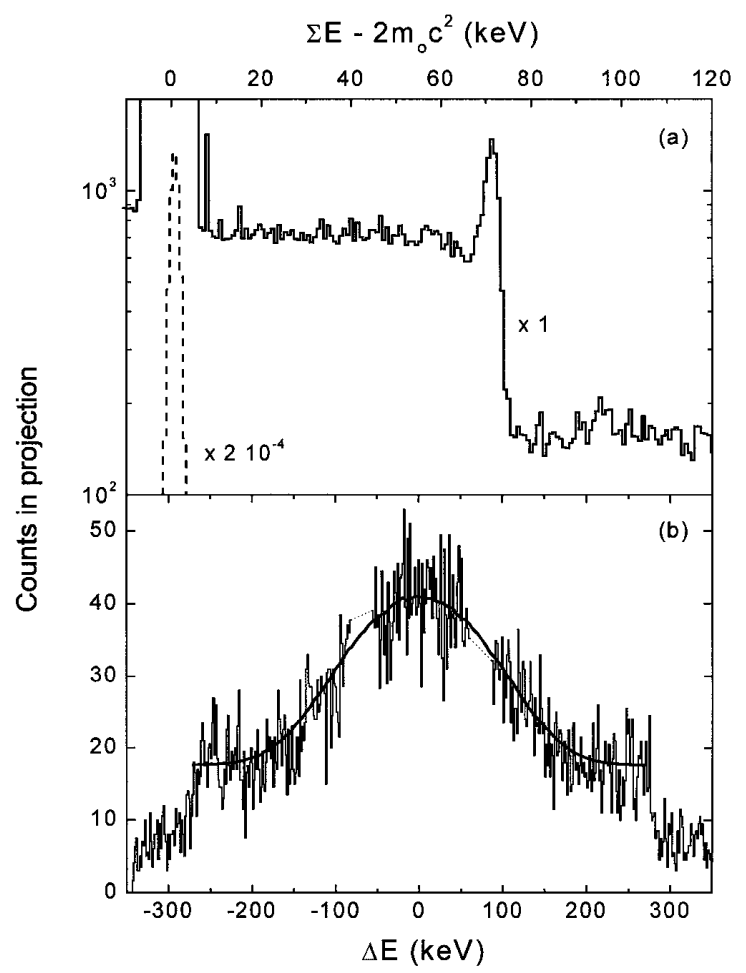

FIG. 2. Projection results in the difference energy direction (a) and the sum energy direction (b) from the data in Fig. 1. Part (a) consists of an integration across the full $\Delta E$ width after the ridge elimination. The dashed line is the thermal peak part reduced by a factor of $2 \times 10^{-4}$. In part (b) the integration was performed across $10 \mathrm{keV}$ centered at $71.6 \mathrm{keV}$ $\sum E$. The smooth line is based on Eq. (2) folded with the angular resolution of the system. 
The total momentum of the electron-positron pair can point towards one of the detectors when the positron scatters prior to annihilation. In the case of large angle elastic scattering the maximum possible energy shift among the photons $\Delta E_{\max }$ can be observed. $\Delta E_{\max }$ can be calculated from Eq. (2).

$$
\Delta E_{\max }= \pm \sqrt{T\left(T+m_{0} c^{2}\right)} .
$$

At $T=71.6 \mathrm{keV}$ the $\Delta E_{\max }$ is $\pm 276 \mathrm{keV}$, consistent with the steps in the $\Delta E$ spectrum. No steps were observed in spectra from the bare $\mathrm{C}$ foil and other low- $Z$ targets like $\mathrm{Al}$ and Ni because the probability for elastic large-angle scattering followed by annihilation is much lower.

To observe annihilations with inner shell electrons from gold, the $\sum E$ spectra from the $\mathrm{C}$ foil before and after $\mathrm{Au}$ coating were compared. An error function (the detector resolution is the width of the step) was fitted to the inelastic contribution under the TQAF peak in the $\sum E$ spectrum and subtracted. Figure 3 shows the results for bare $\mathrm{C}$ and $\mathrm{Au}$ on $\mathrm{C}$ normalized to equal positron illumination. Annihilations with inner gold shell electrons significantly broaden the $\mathrm{Au}$ on the $\mathrm{C}$ line on the low energy side. A fit of a single Gaussian to the C-foil peak is centered at $71.59 \pm 0.02 \mathrm{keV}$ with a FWHM of $2.23 \pm 0.05 \mathrm{keV}$. This is in good agreement with the beam energy and the combined detector resolution. Annihilations with core electrons will appear as additional peaks in the $\sum E$ spectrum. While no separate peaks appear above the statistical scatter of the gold-carbon foil data, the TQAF peak is significantly broadened on the low energy side. Gold $M$-shell electrons are bound with $2.57 \mathrm{keV}$. The fit if a single peak to the gold on carbon data yields a centroid at $70.65 \pm 0.07 \mathrm{keV}$ and $4.26 \pm 0.16 \mathrm{keV}$ FWHM clearly shifted below the beam energy and much broader. In a two-Gaussian fit the second peak is $-2.8 \pm 0.2 \mathrm{keV}$

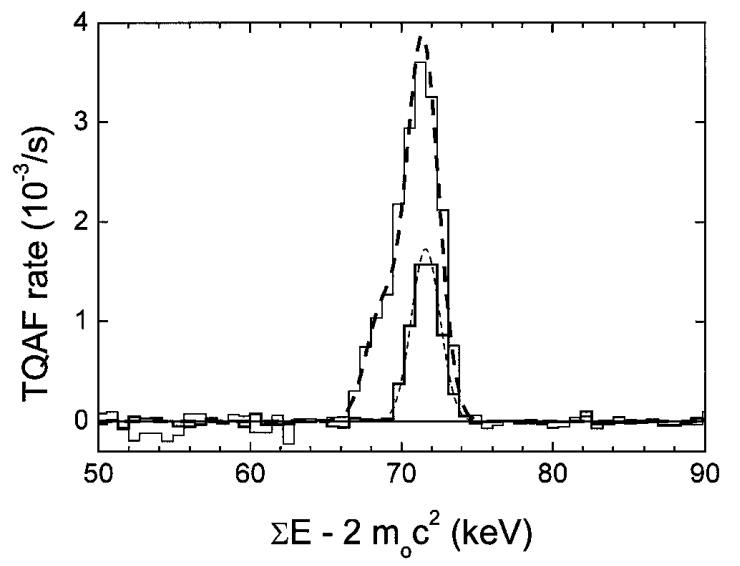

FIG. 3. TQAF peaks from the gold on carbon-foil data and the carbon foil alone (smaller peak) as a function of detected sum energy less the rest mass. The dashed lines are the results of the fits with two and one Gaussian, respectively. The error in the data is on the order of the variations in counts in the energy range from 50 to $64 \mathrm{keV}$. shifted from the TQAF peak and $2.6 \pm 0.2 \mathrm{keV}$ wide in excellent agreement with the $M$-shell binding energy of gold. The results are included as dashed lines in Fig. 3. With a higher energy and higher intensity positron beam TQAF measurements could become a new tool to directly detect annihilations with core electrons as a function of positron energy [19-21].

Additional data were taken at 10,20,30, and $50 \mathrm{keV}$ on the bare carbon foil to examine the energy dependence. In this energy range the positron intensity varies less than $5 \%$ as determined by dumping the beam onto a thick Be target. Positron backscattering from $\mathrm{Be}$ is low and did not have to be taken into account $[22,23]$. The areas under the TQAF peaks in the $\Delta E$ spectra, normalized to constant positron flux, are plotted in Fig. 4 as a function of the inverse of the incident positron velocity in units of $c$. Statistical error bars of 1 standard deviation are included. Also shown is a best fit of the $c / v$ dependence to the data with $\sigma_{\text {rel }}=\sigma_{0}^{*}(c / v-1)+B$. The linear function was written such that the intercept at zero occurs at the maximum possible velocity $c / v=1$. The fit results are a residual constant background $B=(2.8 \pm 10) \times$ $10^{-3} \mathrm{~s}^{-1}$, and $\sigma_{0}=(6.17 \pm 0.14) \times 10^{-3} \mathrm{~s}^{-1}$. The fit, shown as a solid line in the region of the data and dashed line for high velocities near the speed of light, agrees well with the data.

In conclusion, we have measured relative cross sections for two-quantum annihilation of positrons in flight. Annihilations with gold $M$-shell electrons could be isolated from the TQAF line. For carbon the data are in good agreement with the predicted $c / v$ dependence of the cross section in the energy range from 10 to $71.6 \mathrm{keV}$. This experimental technique is applicable to a variety of questions. In combination with positron channeling it can be used to observe magnetic structures in a solid on an atomic scale $[24,25]$. The two-detector spectra provide direct information about the positron velocity, path length traversed in



FIG. 4. TQAF event rate as a function of the inverse velocity of the positrons impinging on the plain carbon foil. The line is a fit of the $c / v$ dependence to the data. Statistical error bars are shown. Towards larger velocities $(v / c \rightarrow 1)$, where the approximation breaks down, the line is dashed. 
the target, and angular distribution at the instant of annihilation. A careful analysis of the bowl may lead to a new way to investigate the energy loss process of positrons in matter. While TQAF events can usually be safely ignored during the analysis of positron annihilation spectroscopy data, in some special applications it is no longer safe to do so. Systematic effects may become significant in techniques like age-momentum spectroscopy [26].

This work was supported in part by the Department of Energy under Contract No. W-7405-ENG-48. Funding for A.W.H. and J.A.G. was provided by the National Science Foundation under Contract No. DMR-9623610.

[1] A. Perkins and J.P. Carbotte, Phys. Rev. B 1, 101 (1970).

[2] P. Kubica and A. T. Stewart, Phys. Rev. Lett. 34, 852 (1975).

[3] S. A. Colgate and F.C. Gilbert, Phys. Rev. 89, 790 (1953).

[4] J. B. Gerhart, B.C. Carlson, and R. Sherr, Phys. Rev. 94, 917 (1954).

[5] H. W. Kendall and M. Deutsch, Phys. Rev. 101, 20 (1956).

[6] E. Malamud and R. Weil, Helv. Phys. Acta 33, 991 (1960).

[7] F. D. Seward, C. R. Hatcher, and S. C. Fultz, Phys. Rev. 121, 605 (1961).

[8] P. Kilian, Z. Phys. 164, 416 (1961).

[9] B. Adeva, Phys. Lett. B 250, 199 (1990).

[10] M.Z. Akrawy et al., Phys. Lett. B 257, 531 (1991).

[11] E. Fernandez et al., Phys. Rev. D 35, 1 (1987).

[12] S. H. Connell, R. W. Fearick, R. F. A. Hoernlé, E. SiderasHaddad, and J. P. F. Sellschop, Phys. Rev. Lett. 60, 2242 (1988).
[13] T. J. Radcliffe, T. K. Alexander, G. C. Ball, H. C. Evans, J. R. Leslie, H. B. Mak, W. McLatchie, P. Skensved, and A. T. Stewart, Phys. Rev. C 42, R2275 (1990).

[14] W. H. Trzaska, H. Dejbakhsh, S. B. Dutta, Q. Li, and T. M. Cormier, Phys. Lett. B 269, 54 (1991).

[15] P. J. Schultz and K. G. Lynn, Rev. Mod. Phys. 60, 701 (1988).

[16] J. R. MacDonald, K. G. Lynn, R. A. Boie, and M.F. Robbins, Nucl. Instrum. Methods 153, 189 (1978).

[17] P. Asoka-Kumar, M. Alatalo, V. J. Ghosh, A. C. Kruseman, B. Nielsen, and K. G. Lynn, Phys. Rev. Lett. 77, 2097 (1996).

[18] P. A. M. Dirac, Proc. Cambridge Philos. Soc. 26, 361 (1930).

[19] Shin Ito, S. Shimmizu, T. Kawaratani, and K. Kubota, Phys. Rev. A 22, 407 (1980).

[20] N. W. Lennard, P. J. Schultz, and G. R. Massoumi, Nucl. Instrum. Methods Phys. Res., Sect. B 33, 128 (1988).

[21] P. J. Schultz and J. L. Campbell, Phys. Lett. 112A, 316 (1985).

[22] J. Makinen, S. Palko, J. Martikainen, and P. Hautojarvi (private communication).

[23] G. R. Massoumi, N. Hozhabri, W. N. Lennard, and P. J. Schultz, Phys. Rev. B 44, 3486 (1991).

[24] R. Haakenaasen, L. V. Hau, J. A. Golovchenko, J.C. Palathingal, J. P. Peng, P. Asoka-Kumar, and K. G. Lynn, Phys. Rev. Lett. 75, 1650 (1995).

[25] L. V. Hau, J. A. Golovchenko, R. Haakenaasen, A. W. Hunt, J. P. Peng, J. C. Palathingal, K. G. Lynn, M. Weinert, and J. C. Palathingal, Nucl. Instrum. Methods Phys. Res., Sect. B 119, 30 (1996).

[26] W. Bauer, K. Maier, J. Major, H. E. Schaefer, A. Seeger, H. D. Carstanjen, W. Decker, J. Diehl, and H. Stoll, Appl. Phys. A 43, 261 (1987). 